\title{
Die vertikale Verteilung von Ciliaten im Stygorhithral der Fulda (Beitrag zur Kenntnis mesopsammaler Ciliaten in Fließgewässern)
}

\author{
Von \\ Günther LÜPKES*
}

\begin{abstract}
SUMMARY
The vertical distribution of stygorhithral ciliates in the Fulda-River (Contribution to the knowledge of mesopsammal ciliates in running freshwater)

19 species of ciliates were found in interstitial biotopes of the Fulda Headwater. Two of these were new: Haplocaulus hengsti, n.sp. and Epistylis rotti, n.sp. (Peritrichida).

The number of ciliate species decreases from the surface down to deeper layers; in a depth of $50 \mathrm{~cm}$, only three species were found: Haplocaulus hengsti and Epistylis rotti and another small ciliate Trachelophyllum apiculatum. There was little detritus in this layer, and so, the abundance of the ciliates was low. On the other hand, there is little predation in deeper layers of stygorhithral sand and gravel. Small, long and thin ciliates seem to be specially adapted to life in deeper regions of the stygornithral Stygorhithral ciliates are related to custygal species with respect to several characteristics of their morphology and general biology.
\end{abstract}

\section{EINLEITUNG}

Der Schwerpunkt faunistisch-ökologischer und systematischer Untersuchungen sowohl des Grundwassers als auch mesopsammaler Lückensysteme in Fließgewässern lag bisher fast ausschließlich auf der Metazoenfauna. Dies mag einerseits an der vergleichsweise relativ geringen Besiedlungsdichte einzelliger Organismen in interstitiellen Lückensystemen, und der sich hieraus ergebenden Untersuchungsschwierigkeiten liegen. Zum andern ist aber auch die Bedeutung der Protozoen als echter Bestandteil der Interstitialbiozönose bisher vollkommen unterschätzt worden. A. Ruttner Kolisko (1956, S. 425) betont daher mit Recht, daß u.a. die Beschreibung subterraner Ciliaten als systematisches Neuland anzusehen sei, das immer noch eines Bearbeiters harre! Es war deshalb das Ziel dieser Untersuchung, nach meinen Befunden aus dem Eustygal der Fulda-Talaue einerseits zu einer systematischen Bestandsaufnahme stygorhith-

* Limnologische Flußstation des Max-Planck-Instituts für Limnologie D. 6407 Schlitz. 
raler Ciliaten zu gelangen und danach ökologische Befunde dieser Organismen mit "echten" Grundwasserformen zu vergleichen. Als Studienobjekt für die vorliegenden Untersuchungen diente die Fulda, ein Mittelgebirgsbach der Rhön, der schon oft Gegenstand limnologischer Untersuchungen gewesen ist. Die genaue Untersuchungsstelle liegt oberhalb des Ortes Gersfeld (s. geol. Karte)

\section{METHODIK}

Die Ciliaten wurden mit Hilfe eines Entnahmegerätes aus verschiedenen Tiefen des Bachschotters zutage befördert. Das Gerät war im Bachschotter eingegraben, wodurch periodische Untersuchungen der Fauna ermöglicht wurden, ohne die Biozönose unnötig zu stören. (nähere Einzelheiten s. Husmann 1976.) Für die Untersuchungen wurden Proben aus Tiefen von 10, 25 und $50 \mathrm{~cm}$ entnommen. So konnten Aussagen über die Abundanz der Ciliaten in den einzelnen Tiefen gemacht werden, und darüber hinaus konnte festgestellt werden, welche der Organismen in tiefere Regionen vorzudringen vermochten. Die Entnahme erfolgte mit Hilfe einer mechanischen Pumpe und einer Wulff'schenFlasche. Nach der Entnahme wurden die Protozoen sicherheitshalber gleich in einen Thermosbehälter und darüber hinaus noch in eine Kühltasche gebracht, um Temperaturschwankungen zu vermeiden. Im Labor erfolgte die Untersuchung der Ciliaten sofort, um auch empfindliche Formen erfassen zu können, die sonst sicher abgestorben wären. Das Material mußte in jedem Fall lebend bestimmt werden, da besonders empfindliche Formen nicht fixiert werden konnten,

\section{DER BIOTOP}

Das untersuchte Bachschotterbecken der Fulda ist an der gesamten Untersuchungsstelle von Laubholzbewuchs umgeben. Hieraus resultiert eine starke Infiltration allochthonem Materials in das Substrat, was für die weiteren Überlegungen sehr wichtig war. Das Substrat des untersuchten Bachabschnittes besteht aus Sand und Kiespartikeln unterschiedlicher Korngröße, wobei in zunehmender Tiefe das Lückensystem sich verengt. Der Detritus, der die Nahrungsgrundlage der im Stygorhithral lebenden Protozoen bildet, besteht aus allochthonem und autochthonem Material, wobei der Detritusgehalt in zunehmender Tiefe abnimmt. Durch diese Veränderung der Bedingungen - hinzu kommt die bereits erwähnte Verengung des Lückensystems - eignen sich besonders die tieferen Regionen dieses Biotopes nur für speziell angepaßte vornehmlich kleine Organismen als Dauerlebensraum. Es kann daher mit Recht gesagt werden, daß zumindest die tieferen Regionen des Bachschotters einen weitgehend abgeschirmten Biotop eigener Prägung darstellen.

\section{DIE BIOZÖNOSE}

Die Untersuchungsstelle erwies sich als Fundstätte der verschiedensten Kleinorganismen, wobei die Metazoen in dieser Arbeit unberücksichtigt bleiben, und daher auch nicht weiter erwähnt werden. Das Material enthielt reichliches Protozoenmaterial vieler Ordnungen. Es wurden u.a. Testaceen, Flagellaten, Heliozoen gefunden, die aber nicht näher bestimmt oder weiterer Bearbeitungen 
zugeführt wurden. Es fanden sich bei der Bearbeitung der Proben 19 verschiedene Ciliatenarten. Zwei der erfaßten Protozoen sind für die Wissenschaft neu und werden als Haplocaulus hengsti, sp.n. und Epistylis rotti, sp.n. beschrieben. Durch das Auffinden der beiden Holophryiden Trachelophyllum apiculatum (chilense) und Trachelophyllum clavatum im selben Biotop, konnte die umstrittene Kernsystematik beider Arten geklärt und eine sichere Abgrenzung dieser Formen gegeneinander erreicht werden. Weiterhin ist wesentlich, daß nur drei von neunzehn erfaßten Ciliatenformen $50 \mathrm{~cm}$ in das Stygorhithral der Fulda vorzudringen vermochten. Wesentliche Hindernisfaktoren hierfür sind Form, Flexibilität und die unterschiedlichen Lebensansprüche der Ciliaten, letztenendes aber auch die Abnahme des Nahrungsangebotes in zunehmender Tiefe. Auf dieses Problem wird jedoch in der Diskussion noch eingegangen werden; zunächst steht die Besprechung der wichtigsten Ciliatenformen an.

\section{ÖKOLOGISCHE UND SYSTEMATISCHE BEFUNDE}

1. Haplocaulus hengsti, sp.n.

Meßdaten: Plasmàkörper; Länge: $79 \mu \mathrm{m}$; Breite: $49 \mu \mathrm{m}$; Stiellänge: $73 \mu \mathrm{m}$.

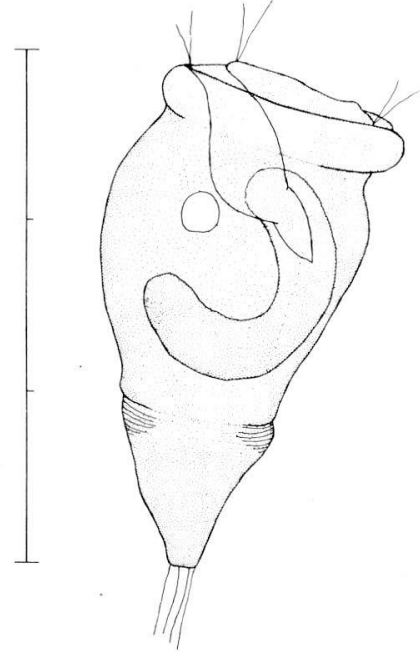

Fig. 1. Haplocaulus hengsti, sp. n. (Peritricha, Zoothamniidae)

Haplocaulus hengsti gehört zur Familie der Zoothamniiden innerhalb der kontraktilen Peritrichen. Der Plasmakörper des Tieres ist sehr hyalin und weist keine besonderen Strukturmerkmale auf. Kennzeichnend und besonders auffallend ist der ungewöhnlich breite, wurstförmige Makronucleus, der etwa senk- 
recht im Körper des Epistyliden liegt. Weiterhin ist eine deutliche Einschnürung des letzten Körperdrittels der Haplocaulus bemerkbar. Erwähnenswert ist noch die seitliche Noppe auf dem Diskus (s.Abb.), die deutlich hervortritt.

2. Epistylis rotti, sp.n.

Meßdaten: Plasmakörper; Länge: $48 \mu \mathrm{m}$; Breite: $27 \mu \mathrm{m}$.

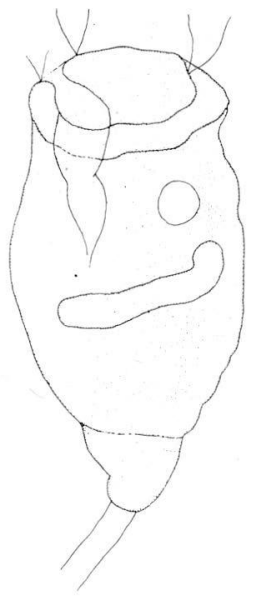

Fig. 2. Epistylis rotti, sp.n. (Peritricha, Epistylidae)

Epistylis rotti gehört zur Gruppe der akontraktilen Peritrichen und zur Familie der Epistyliden. Da ich diese Form bisher nur wenige Male im Detritus antraf, möchte ich sie hier nur vorläufig anführen. Das Peritrich besitzt einen gebogenen Makronucleus, der an einem Ende etwas gestaucht ist. Der Plasmakörper weist im unteren Drittel eine deutliche Querfaltung auf; der Stiel ist kurz, wobei jedoch eine gewisse Variationsbreite nicht auszuschließen ist. Epistylis rotti lebt wie Haplocaulus hengsti in $50 \mathrm{~cm}$ Tiefe des untersuchten Bachschotterabschnittes. Die beiden Peritrichen wurden ausschließlich in dieser Tiefe angetroffen; in anderen Schichten waren sie nicht anzutreffen. Ihre Nahrungsgrundlage bildet der mit Bakterien behaftete Detritus.

3. Trachelophyllum apiculatum var. chilense, Bürger 1906, Kahl 1935. Dieses durch seine stygophile Lebensweise recht bemerkenswerte Infusor fand sich zwischen Sandkörnchen $25 \mathrm{~cm}$ tief im Stygorhithral der Fulda. Wegen der ausgeprägten Variationsbreite der Trachelophyllum-Formen $(30-100 \mu \mathrm{m})$ ist die Bestimmung oft schwierig. Ein sicheres Erkennungsmerkmal der Tr. apiculatum ist jedoch die seitliche Verschmelzung bei der Konjugation. Trachelo- 
phyllum-Arten verschmelzen sonst nur polar. Über die Variationsbreite der Trachelophyllum apiculatum var. chilense verweise ich auf meinen Aufsatz im Mikrokosmos, 1973, so daß ich mir hier nähere Einzelheiten ersparen möchte. Durch die Flexibilität und die geringe Größe des Plasmakörpers ist Tr. apiculatum gut an subterrane Verhältnisse angepaßt. Die Nahrung des Infusors ist recht vielseitig. Hauptnahrung scheint der Detritus zu sein, aber auch Aas wurde nicht verschmäht; so fand sich Trachelophyllum apiculatum zuweilen an und in abgestorbenen Cyclopoiden.

\section{Trachelophyllum clavatum, Stokes 1886}

Hier handelt es sich eine betont kleine Form. (Gr. $100 \mu \mathrm{m}$ ), normale Individuen etwa $180-200 \mu \mathrm{m}$ ). Tr. clavatum fand sich zusammen mit $T r$. apiculatum bis in eine Tiefe von $25 \mathrm{~cm}$. Der einfache Kern, einziger morphologischer Unterschied zu Tr. apiculatum, ließ oft Zweifel am Bestehen dieser Art deutlich werden. Da sich jedoch beide Trachelophyllum-Arten ständig zusammen im Bachschotter fanden, ließ sich die Konstanz des einfachen Kernes bei Tr. clavatum bestätigen, und ich lasse die bisherigen Bedenken in dieser Sache fallen. Übrigens verschmilzt $T r$. clavatum bei der Konjugation polar, was eine sichere Unterscheidung, auch bei Nichtberücksichtigung des Kernes ermöglicht. Die Ernährung der Tr. clavatum besteht aus Detritus. Aus der folgenden Tabelle wird die Verteilung der einzelnen erfaßten Ciliaten in den verschiedenen Tiefen anschaulich:

Art: $\quad$ Tiefe: (in $\mathrm{cm}$ )

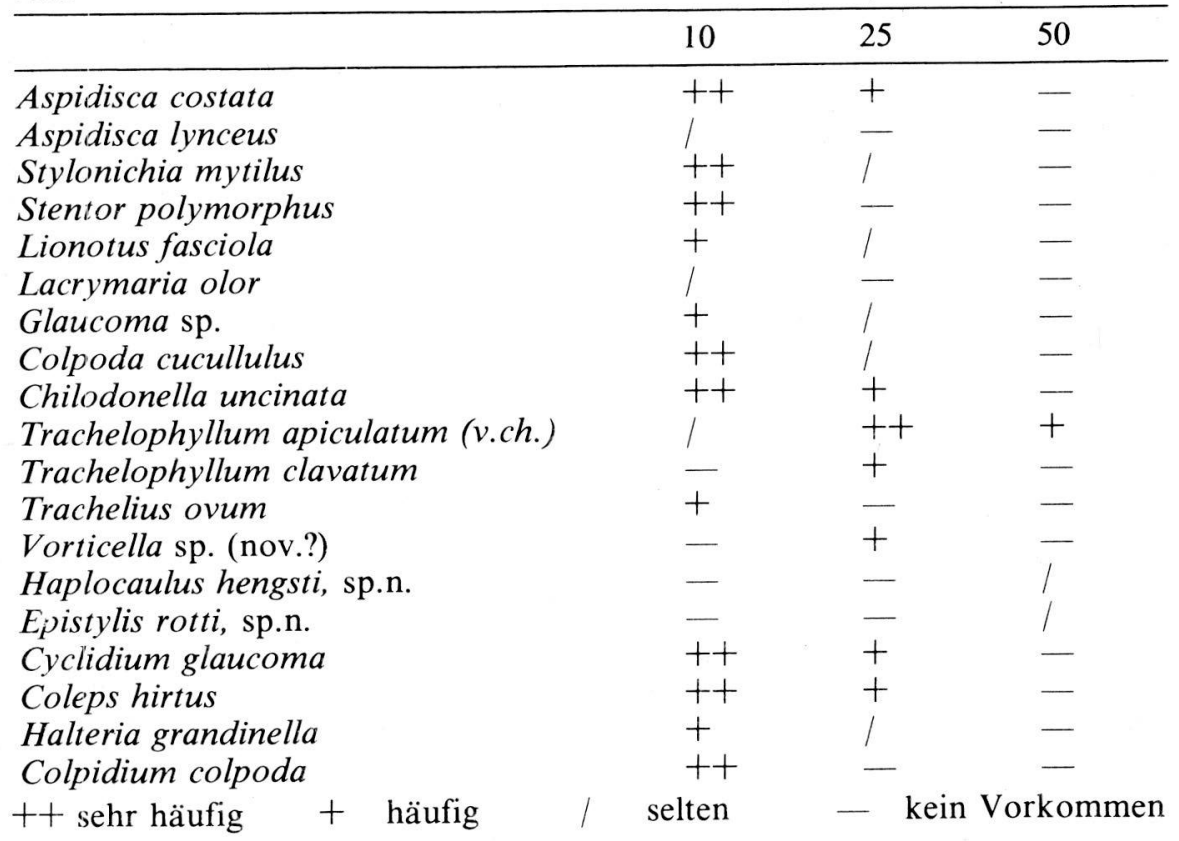




\section{DISKUSSION}

Durch die dargestellten ökologischen und systematischen Befunde wird deutlich, daß das Stygorhithral von Bergbächen ein sehr wichtiges und ergiebiges Refugium für Protozoen zahlreicher Ordnungen darstellt, das bei bisherigen Untersuchungen nur wenig Beachtung fand. Bei dieser Serienuntersuchung bestätigte sich, daß nur Organismen mit geigneter Körpergröße, Form und Flexibilität in tiefere Regionen des Intergranularwassers von Bachschotterbecken vorzudringen vermögen. Es ergeben sich weiterhin bemerkenswerte Paralellen zu den vorhergehenden Grundwasseruntersuchungen in der FuldaTalaue bezüglich der Ciliaten: Mit zunehmender Tiefe des untersuchten Biotops nimmt auch der Einfluß terrestrischer und oberflächenaquatischer Infiltrationen ab. Demzufolge verringert sich auch die Besiedlungsdichte der Ciliaten, da das Nahrungsangebot in zunehmender Tiefe geringer wird. Während bei der Durchmusterung von Proben aus $10 \mathrm{~cm}$ Tiefe noch eine Detritusschwemme von fast durchweg groben Pflanzenpartikeln vorhanden war, bestand der Detritus in $50 \mathrm{~cm}$ Tiefe durch die Fulterwirkung des Sandes nur noch aus feingeriebenen Teilchen. Auch fanden sich nur wenige Ciliaten und es zeigte sich eine tiefentypische Artzusammensetzung. Die Beobachtungen zeigen weiterhin, daß gerade der Ciliatenfauna mesopsammaler Biotope eine Hauptrolle bei der Reinigung der Intergranularräume zukommt. Gerade die aasfressenden Trachelophyllum-Arten demonstrieren eine Notwendigkeit ihrer Anwesenheit, denn ohne die Tätigkeit dieser Organismen würden sich häufiger gefährliche Fäulnisprozesse im Intergranularbereich zeigen. Aus diesen ökologischen Befunden läßt sich erkennen, daß auch subterranen Ciliaten Indikatoren für den jeweiligen Zustand mesopsammaler Biotope darstellen. (z.B. Korngröße Verschmutzung). Die Verschmutzungsempfindlichkeit der Epistylis subterranea, einem reinen Grundwasserperitrich würde diese These stützen. Aus der Verteilung der einzelnen Ciliatenformen in den verschiedenen Schottertiefen läßt sich vorläugif lediglich eine Einteilung der Organismen in oberflächennahe, stygoxene Ciliaten und stygophile Formen vornehmen. Es müssen jedoch noch umfangreiche Untersuchungen durchgeführt werden, die eine allgemeingültige Einteilung subterraner Ciliaten rechtfertigen.

\section{ZUSAMMENFASSUNG}

19 Ciliatenarten wurden im Interstitialbereich der oberen Fulda gefunden. Zwei von diesen sind neu: Haplocaulus hengsti, n.sp. und Epistylis rotti, n.sp. (Peritricha)

Die Anzah! der Ciliaten nimmt von der Oberfläche nach der Tiefe zu ab; in einer Tiefe von $50 \mathrm{~cm}$ fanden sich nur drei Arten, Haplocaulus hengsti, Epistylis rotti und eine andere kleine Form der Trachelophyllum apiculatem. In dieser Schicht gab es nur wenig Detritus, demgemäß war die Besiedlungsdichte der Ciliaten gering. In tieferen Regionen gibt es andererseits Schutzmöglichkeiten für die Ciliaten. Kleine, längliche und schmale Formen von Ciliaten scheinen dem Leben in tieferen Interstitialregionen speziell angepaßt zu sein. Stygorhithrale Ciliaten zeigen eine enge Verwandtschaft zu eustygalen Formen, da sich in mehreren morphologischen und ökologischen Befunden Paralellen zeigen. 


\section{LITERATUR}

HAMMAN, I. (1953): Ökologische und biologische Untersuchungen an Süßwasserperitrichen. Arch. Hydrobiol. 46: 177-228.

HUSMANN, S. (1970): Weitere Vorschläge zur Klassifizierung subterraner Biotope und Biozönosen der Süßwasserfauna. - Int. Rev. ges. Hydrobiol. 55: 115-129.

(1973): Versuche zur Erfassung der vertikalen Verteilung von Organismen und chemischen Substanzen im Grundwasser von Talauen und Terrassen - Methoden und erste Befunde (im Druck).

KAHL, A. (1931): Wimpertiere oder Ciliata, Bd. 1 u. 2 Dahl - Die Tierwelt Deutschlands.

RUTTNER-KOLISKO, A. (1956): Der Lebensraum des Limnopsammals - Verh. Dt. Zool. Ges. in Hamburg: 422-427.

(1953): Psammonstudien I. Heft 3: 130-161 - Math. naturwiss. Kl., Abt. I 162.

LÜPKES, G. (1973): Drei neue stygobionte Peritrichen aus dem interstitiellen Grundwasser Arch. Hydrobiol. 73, 394-402.

(1974): Ein Wimpertier aus Zapfhahnbärten - Mikrokosmos, Heft 7, Juli 1974. 